Bangladesh J. Bot. 49(4): 967-974, 2020 (December)

\title{
EFFECTS OF DIFFERENT SOURCES OF LIGHT AS NIGHT BREAK ON GROWTH CHARACTERISTICS OF KOREAN CHRYSANTHEMUM (CHRYSANTHEMUM MORIFOLIUM RAMAT.) GENOTYPES
}

\author{
RanjIT Singh* and Madhu Bala \\ Department of Floriculture and Landscaping, Punjab Agricultural \\ University, Ludhiana-141004, India
}

Keywords: Chrysanthemum, Night break, Sources of light, LED light

\begin{abstract}
Longer photoperiods in the form of night break (NB) were applied in chrysanthemum to inhibit the flower bud differentiation for regulating the production and supply in the market. The present study reports the effect of three sources of light (Incandescent bulbs, compact fluorescent lights (CFL) and light emitting diodes-LED) as NB treatment on ten genotypes of Korean chrysanthemum for two years i.e. 2015 and 2016. The NB treatment with LED lights resulted in increased internodal length $(8.20$ and $9.00 \mathrm{~mm})$, partitioning coefficient (30.50 and 31.50), number of stomata per unit area (56.08 and 56.87), anthocyanins (8.99 and $9.16 \mathrm{mg} / \mathrm{g}$ ) and carotenoids (28.11 and $28.39 \mathrm{mg} / \mathrm{g}$ ). However, leaf thickness, petiole length, leaf area index, and leaf weight ratio were affected non-significantly. But petiole girth was recorded maximum (1.22 and 1.49 $\mathrm{mm}$ ) under incandescent bulbs which was at par with LED and significantly higher than CFL.
\end{abstract}

\section{Introduction}

Korean chrysanthemum (Chrysanthemum morifolium Ramat.) belonging to family Asteraceae is the commercial flower which is grown for cut flowers, loose flowers, pot mum as well as bedding plant. Singh and Bala (2018) and Thakur and Grewal (2019) have demonstrated the benefits of using supplementary light during the winter periods to raise the levels of visible radiation for the production of cut flowers and pot plants. Chrysanthemum being a short-day plant requires less than $131 / 2 \mathrm{hrs}$ of photoperiods for the development of flower buds (Kofranek 1992). The photoperiods in chrysanthemum may be increased by giving night break (NB) treatment which is widely used in production of chrysanthemum by applying incandescent lamps to inhibit flowering thereby forcing the plant to remain in vegetative phase. For NB treatment various sources of light like high pressure sodium lamp, incandescent bulbs, fluorescent tube lights etc. are being used in horticulture for photoperiodic control as well as for production purpose. New sources of light for photoperiodic manipulations for horticultural applications, intending to replace high pressure sodium lamps or fluorescent tubes are currently being introduced in the market. Special attention is being devoted to light emitting diodes (LED) technology, which are generally assumed to be more efficient for driving photosynthesis to optimum range (Bergstrand and Schussler 2012). As solid-state devices, they are more robust than traditional lamps with fragile filaments, electrodes, or gas-filled, pressurized lamp enclosures. Since LEDs can be manufactured to be wavelength selectable, absorption peaks of plant pigments or action spectra of leaves can be matched by narrow-spectrum LED emitters, thereby maximizing quantum efficiencies and energy savings (Massa et al. 2006). Morrow (2008) also highlighted the advantages of LED lighting including the ability to control spectral quality for improving photosynthetic efficiency, the ability to produce high light levels with low radiant heat, and operating capabilities that are helpful to reduce power use. Therefore, keeping this in view the effect of different sources of light on physiological attributes which directly or indirectly contribute to flower production, the present

*Author for correspondence: <ranjit_flori@pau.edu>. 
study was undertaken to compare the effect of conventional sources of light for NB treatment in Korean chrysanthemum.

\section{Materials and Methods}

The experiments were carried out at Department of Floriculture and Landscaping, Punjab Agricultural University, Ludhiana during 2015 and 2016. Ten genotypes of chrysanthemum belonging to Korean type, namely Banglori Local, Boris Becker, Reagan White, Kelvin Mandarin, NBRI Sunil, Reagan Emperor, Otome Pink, Jaya, Punjab Shyamli and Yellow Delight, were raised through the rooting of terminal cuttings during end of June to mid-July. The rooted cuttings were transplanted in the pots of $20 \mathrm{~cm}$ diameter containing mixture of garden soil and well rotten Farm Yard manure $(2: 1)$ along with diammonium phosphate @ $1 \mathrm{~kg}$ per cubic feet. The pots were placed under longer photoperiodic conditions created as NB, from 15th August to 31st October, as day length starts receding less than $14 \mathrm{hrs}$ after 15th August. The NB treatment was provided by using three sources of light viz., incandescent bulbs, CFL and LED lamps of approximately same wattage $(25 \mathrm{~W})$ for two hrs from 10.00 p.m. to 12.00 midnight daily. The plants were shifted to natural day length on 1 st November. The varieties were subjected to pinching as per the standard practice. The observations were recorded on vegetative and at the time of flowering.

Partitioning coefficient: PC was calculated at commercial harvesting stage, as follows:

$$
\frac{\text { Dry weight of flowers }}{\text { Dry weight of plants }} \times 100
$$

Leaf area index (LAI) (Gardener et al. 2003) LAI was calculated as follows:

$$
\mathrm{LAI}=\frac{\text { Total leaf area per plant } \times \text { number of plants } / \mathrm{m}^{2}}{\text { Surface area of land }}
$$

Leaf weight ratio: LWR was calculated as follows:

$$
\text { LWR = Dry weight of leaves/dry weight of plant. }
$$

To calculate number of stomata per unit area, Olympus trinocular microscope with a magnification of $100 \mathrm{X}$ was used. Numbers of stomata were counted on three randomly chosen areas on the leaf surface $\left(1 \mathrm{~mm}^{2}\right.$, middle of leaf on both sides of the main vein) on abaxial and adaxial surfaces of the leaves.

Experiment was laid out with three replications consisting five pots each, in completely randomized design. Statistical analysis was performed using SAS software and the means were compared using DMRT at 5\% level of significance.

\section{Results and Discussion}

The plants under LED light exhibited highest internodal length during 2015 and 2016 (8.20 and $9.00 \mathrm{~mm}$, respectively). However, the lowest internodal length was recorded in CFL for the both years ( 7.70 and $7.89 \mathrm{~mm}$, respectively) which was at par with incandescent bulb (Table 1). Among the genotypes, on averaging across the different light sources, significantly the highest internodal length (10.83 and $11.02 \mathrm{~mm}$ ) was observed in genotype Punjab Shyamli during 2015 and 2016, respectively. Whereas, the minimum internodal length was observed in genotype Banglori Local $(4.53 \mathrm{~mm} 4.74 \mathrm{~mm}$ ) during the same period of study. The increased internodal length contribute to enhanced flower stem length which is a useful quality parameter for fetching higher price for the cut flowers. Sarkka et al. 2017 have also found the incremental increase in 
internodal length and water use efficiency while comparing the effects of LED and high-pressure sodium (HPS) on cucumber plant morphology and yield but the results obtained, are however, in contradiction with those as reported by Kumar and Singh (2017), who have reported nonsignificant effect of extended photoperiods on internodal length in chrysanthemum cultivar Zembla using HPS lamps. Further leaf thickness, petiole length, leaf area index and leaf weight ratio were not significantly affected by the sources of the light applied but the average across all

Table 1. Effect of different sources of light as night break on internodal length in Korean chrysanthemum.

\begin{tabular}{|c|c|c|c|c|c|c|c|c|c|}
\hline \multirow{3}{*}{$\begin{array}{l}\text { Sl. } \\
\text { No. }\end{array}$} & \multirow{3}{*}{ Genotype } & \multicolumn{8}{|c|}{ Internodal length (mm) } \\
\hline & & \multicolumn{3}{|c|}{2015} & \multirow[b]{2}{*}{ Mean } & \multicolumn{3}{|c|}{2016} & \multirow[b]{2}{*}{ Mean } \\
\hline & & $\begin{array}{l}\text { Incande- } \\
\text { scent bulb }\end{array}$ & CFL & LED & & $\begin{array}{l}\text { Incande- } \\
\text { scent bulb }\end{array}$ & CFL & LED & \\
\hline 1 & Banglori Local & 2.67 & 1.81 & 9.11 & $4.53^{\mathrm{e}}$ & 2.84 & 2.03 & 9.35 & $4.74^{\mathrm{c}}$ \\
\hline 2 & Boris Becker & 7.41 & 8.10 & 7.95 & $7.82^{\mathrm{c}}$ & 7.61 & 8.30 & 8.19 & $8.03^{\mathrm{ab}}$ \\
\hline 3 & Reagan White & 7.36 & 6.67 & 8.51 & $7.51^{\mathrm{c}}$ & 7.61 & 6.92 & 8.78 & $7.77^{\mathrm{b}}$ \\
\hline 4 & Kelvin Mandarin & 5.37 & 6.00 & 9.69 & $7.02^{\mathrm{cd}}$ & 5.55 & 6.18 & 9.92 & $7.22^{\mathrm{bc}}$ \\
\hline 5 & NBRI Sunil & 8.56 & 8.83 & 1.10 & $6.16^{\mathrm{d}}$ & 8.73 & 9.00 & 7.10 & $8.28^{\mathrm{ab}}$ \\
\hline 6 & Reagan Emperor & 10.75 & 9.55 & 7.62 & $9.31^{\mathrm{b}}$ & 10.91 & 9.72 & 7.80 & $9.48^{\mathrm{ab}}$ \\
\hline 7 & Otome Pink & 7.77 & 6.63 & 8.83 & $7.74^{\mathrm{c}}$ & 8.19 & 6.78 & 8.99 & $7.99^{\mathrm{ab}}$ \\
\hline 8 & Jaya & 7.97 & 7.69 & 11.36 & $9.01^{\mathrm{b}}$ & 8.17 & 7.89 & 11.57 & $9.21^{\mathrm{ab}}$ \\
\hline 9 & Punjab Shyamli & 11.00 & 13.13 & 8.37 & $10.83^{\mathrm{a}}$ & 11.18 & 13.32 & 8.57 & $11.02^{\mathrm{a}}$ \\
\hline \multirow[t]{2}{*}{10} & Yellow Delight & 8.67 & 8.60 & 9.52 & $8.93^{\mathrm{b}}$ & 8.90 & 8.83 & 9.75 & $9.16^{\mathrm{ab}}$ \\
\hline & Mean & $7.75^{\mathrm{b}}$ & $7.70^{\mathrm{b}}$ & $8.20^{\mathrm{a}}$ & & $7.97^{\mathrm{b}}$ & $7.89^{\mathrm{b}}$ & $9.00^{\mathrm{a}}$ & \\
\hline
\end{tabular}

Table 2. Effect of different sources of light as night break on leaf thickness in Korean chrysanthemum.

\begin{tabular}{|c|c|c|c|c|c|c|c|c|c|}
\hline \multirow{3}{*}{$\begin{array}{l}\text { Sl. } \\
\text { No. }\end{array}$} & \multirow{3}{*}{ Genotype } & \multicolumn{8}{|c|}{ Leaf thickness (mm) } \\
\hline & & \multicolumn{3}{|l|}{2015} & \multirow[t]{2}{*}{ Mean } & \multicolumn{3}{|l|}{2016} & \multirow[t]{2}{*}{ Mean } \\
\hline & & $\begin{array}{l}\text { Incande- } \\
\text { scent bulb }\end{array}$ & CFL & LED & & $\begin{array}{l}\text { Incande- } \\
\text { scent bulb }\end{array}$ & CFL & LED & \\
\hline 1 & Banglori Local & 0.64 & 0.76 & 0.90 & $0.77^{\mathrm{a}}$ & 0.81 & 0.98 & 1.14 & $0.98^{\mathrm{a}}$ \\
\hline 2 & Boris Becker & 0.67 & 0.56 & 2.70 & $1.31^{\mathrm{a}}$ & 0.86 & 0.77 & 2.93 & $1.52^{\mathrm{a}}$ \\
\hline 3 & Reagan White & 0.66 & 0.73 & 0.76 & $0.72^{\mathrm{a}}$ & 0.91 & 0.98 & 1.02 & $0.97^{\mathrm{a}}$ \\
\hline 4 & Kelvin Mandarin & 0.90 & 1.00 & 0.99 & $0.96^{\mathrm{a}}$ & 1.05 & 1.18 & 1.23 & $1.15^{\mathrm{a}}$ \\
\hline 5 & NBRI Sunil & 0.82 & 0.88 & 0.99 & $0.90^{\mathrm{a}}$ & 0.99 & 1.05 & 0.99 & $1.01^{\mathrm{a}}$ \\
\hline 6 & Reagan Emperor & 0.66 & 0.83 & 0.89 & $0.79^{\mathrm{a}}$ & 0.82 & 1.00 & 1.07 & $0.96^{\mathrm{a}}$ \\
\hline 7 & Otome Pink & 0.54 & 0.69 & 0.75 & $0.66^{\mathrm{a}}$ & 0.95 & 0.84 & 0.91 & $0.90^{\mathrm{a}}$ \\
\hline 8 & Jaya & 0.55 & 0.66 & 0.72 & $0.64^{\mathrm{a}}$ & 0.75 & 0.86 & 0.94 & $0.85^{\mathrm{a}}$ \\
\hline 9 & Punjab Shyamli & 0.62 & 0.66 & 0.70 & $0.66^{\mathrm{a}}$ & 0.81 & 0.85 & 0.90 & $0.85^{\mathrm{a}}$ \\
\hline 10 & Yellow Delight & 0.41 & 0.51 & 0.55 & $0.49^{\mathrm{a}}$ & 0.64 & 0.74 & 0.78 & $0.72^{\mathrm{a}}$ \\
\hline \multicolumn{2}{|c|}{ Mean } & $0.65^{\mathrm{a}}$ & $0.73^{\mathrm{a}}$ & $0.99^{\mathrm{a}}$ & & $0.86^{\mathrm{a}}$ & $0.93^{\mathrm{a}}$ & $1.19^{\mathrm{a}}$ & \\
\hline
\end{tabular}

Mean values in each column with the same letter are not significantly different at $\mathrm{p}<0.05$ according to DMRT. *Significant at $\mathrm{p}<0.05$. 
Table 3. Effect of different sources of light as night break on petiole length in Korean chrysanthemum.

\begin{tabular}{|c|c|c|c|c|c|c|c|c|c|}
\hline \multirow{3}{*}{$\begin{array}{l}\text { Sl. } \\
\text { No. }\end{array}$} & \multirow{3}{*}{ Genotype } & \multicolumn{8}{|c|}{ Petiole length $(\mathrm{mm})$} \\
\hline & & \multicolumn{3}{|l|}{2015} & \multirow[t]{2}{*}{ Mean } & \multicolumn{3}{|c|}{2016} & \multirow[t]{2}{*}{ Mean } \\
\hline & & $\begin{array}{l}\text { Incande- } \\
\text { scent bulb }\end{array}$ & CFL & LED & & $\begin{array}{l}\text { Incande- } \\
\text { scent bulb }\end{array}$ & CFL & LED & \\
\hline 1 & Banglori Local & 5.42 & 6.42 & 8.40 & $6.75^{\mathrm{g}}$ & 6.66 & 8.04 & 10.01 & $8.24^{\mathrm{g}}$ \\
\hline 2 & Boris Becker & 6.41 & 8.44 & 14.43 & $9.76^{\mathrm{fe}}$ & 6.69 & 8.80 & 14.94 & $10.14^{\mathrm{ef}}$ \\
\hline 3 & Reagan White & 14.92 & 14.53 & 11.60 & $13.68^{b}$ & 15.03 & 14.67 & 11.87 & $13.86^{\mathrm{b}}$ \\
\hline 4 & Kelvin Mandarin & 12.16 & 11.45 & 14.06 & $12.56^{\mathrm{bc}}$ & 12.28 & 11.60 & 14.33 & $12.74^{\mathrm{bc}}$ \\
\hline 5 & NBRI Sunil & 15.75 & 14.44 & 14.27 & $14.82^{\mathrm{a}}$ & 15.93 & 14.73 & 14.63 & $15.10^{\mathrm{a}}$ \\
\hline 6 & Reagan Emperor & 14.72 & 14.38 & 9.68 & $12.93^{b c}$ & 14.90 & 14.67 & 9.99 & $13.19^{\mathrm{bc}}$ \\
\hline 7 & Otome Pink & 9.24 & 9.32 & 10.27 & $9.61^{\mathrm{f}}$ & 9.38 & 9.50 & 10.61 & $9.83^{\mathrm{f}}$ \\
\hline 8 & Jaya & 8.31 & 10.88 & 13.32 & $10.84^{\mathrm{de}}$ & 8.53 & 11.09 & 13.67 & $11.10^{\mathrm{de}}$ \\
\hline 9 & Punjab Shyamli & 14.36 & 14.00 & 7.03 & $11.80^{\mathrm{cd}}$ & 14.52 & 14.24 & 7.35 & $12.04^{\mathrm{cd}}$ \\
\hline 10 & Yellow Delight & 7.01 & 6.67 & 6.38 & $6.69^{\mathrm{g}}$ & 7.10 & 6.79 & 6.60 & $6.83^{\mathrm{h}}$ \\
\hline Mean & & $10.83^{\mathrm{a}}$ & $11.05^{\mathrm{a}}$ & $10.94^{\mathrm{a}}$ & & $11.10^{\mathrm{a}}$ & $11.41^{\mathrm{a}}$ & $11.40^{\mathrm{a}}$ & \\
\hline
\end{tabular}

Table 4. Effect of different sources of light as night break on leaf area index in Korean chrysanthemum.

\begin{tabular}{|c|c|c|c|c|c|c|c|c|c|}
\hline \multirow{3}{*}{$\begin{array}{l}\text { Sl. } \\
\text { No. }\end{array}$} & \multirow{3}{*}{ Genotype } & \multicolumn{8}{|c|}{ Leaf area index } \\
\hline & & \multicolumn{3}{|c|}{2015} & \multirow[b]{2}{*}{ Mean } & \multicolumn{3}{|c|}{2016} & \multirow[b]{2}{*}{ Mean } \\
\hline & & $\begin{array}{l}\text { Incande- } \\
\text { scent bulb }\end{array}$ & CFL & LED & & $\begin{array}{l}\text { Incande- } \\
\text { scent bulb }\end{array}$ & CFL & LED & \\
\hline 1 & Banglori Local & 0.47 & 0.50 & 0.55 & $0.51^{\mathrm{a}}$ & 0.49 & 0.51 & 0.54 & $0.51^{\mathrm{b}}$ \\
\hline 2 & Boris Becker & 0.46 & 0.51 & 0.53 & $0.50^{\mathrm{a}}$ & 0.49 & 0.52 & 0.54 & $0.52^{\mathrm{b}}$ \\
\hline 3 & Reagan White & 0.47 & 0.50 & 0.53 & $0.50^{\mathrm{a}}$ & 0.49 & 0.51 & 0.52 & $0.51^{\mathrm{b}}$ \\
\hline 4 & Kelvin Mandarin & 0.39 & 0.38 & 0.47 & $0.41^{\mathrm{a}}$ & 0.40 & 0.37 & 0.46 & $0.41^{\mathrm{b}}$ \\
\hline 5 & NBRI Sunil & 0.41 & 0.39 & 0.24 & $0.35^{\mathrm{b}}$ & 0.40 & 0.39 & 0.23 & $0.34^{\mathrm{b}}$ \\
\hline 6 & Reagan Emperor & 0.37 & 0.43 & 0.42 & $0.41^{\mathrm{a}}$ & 0.36 & 0.42 & 0.43 & $0.40^{\mathrm{b}}$ \\
\hline 7 & Otome Pink & 0.38 & 0.36 & 0.39 & $0.38^{\mathrm{b}}$ & 0.34 & 0.37 & 0.38 & $0.36^{\mathrm{b}}$ \\
\hline 8 & Jaya & 0.50 & 0.52 & 0.51 & $0.51^{\mathrm{a}}$ & 0.51 & 0.51 & 0.53 & $0.52^{\mathrm{a}}$ \\
\hline 9 & Punjab Shyamli & 0.57 & 0.59 & 0.60 & $0.59^{\mathrm{a}}$ & 0.59 & 0.58 & 0.62 & $0.60^{\mathrm{a}}$ \\
\hline 10 & Yellow Delight & 0.40 & 0.40 & 0.42 & $0.41^{\mathrm{a}}$ & 0.40 & 0.42 & 0.42 & $0.43^{\mathrm{b}}$ \\
\hline Mean & & $0.44^{\mathrm{a}}$ & $0.46^{\mathrm{a}}$ & $0.47^{\mathrm{a}}$ & & $0.45^{\mathrm{a}}$ & $0.46^{\mathrm{a}}$ & $0.47^{\mathrm{a}}$ & \\
\hline
\end{tabular}

Mean values in each column with the same letter are not significantly different at $\mathrm{p}<0.05$ according to DMRT.

*Significant at $\mathrm{p}<0.05$.

the light sources among the various genotypes showed significant difference. The leaf thickness during 2015 and 2016 was recorded maximum $(0.96$ and $1.15 \mathrm{~mm}$, respectively) in Kelvin Mandarin (Table 2) whereas Yellow Delight exhibited minimum leaf thickness $(0.49$ and 0.72 $\mathrm{mm}$, respectively during 2015 and 2016). For both years petiole length was found maximum (14.82 and $15.10 \mathrm{~mm}$, respectively) in NBRI Sunil whereas, Otome Pink exhibited shortest petiole (Table 3). Similar variation was observed for leaf area index with maximum (0.59 and 0.60) in Punjab Shyamli which was at par with Jaya for both the years (Table 4). Night break using LED lights have resulted in highest partitioning coefficient during 2015 and 2016 (30.50 and 31.50, respectively) whereas, it was recorded lowest (28.83 and 29.75) in incandescent bulb (Table 5). 
Genotype Otome Pink exhibited highest partitioning coefficient for both years (43.60 and 44.48). The partitioning coefficient indicates the amount of photosynthetic assimilates utilized for the development of flower buds and flowers (Kaur 2014). Higher values indicate that most of the carbohydrates assimilated through photosynthesis have been used up for the development of the flowers. Leaf weight ratio was recorded maximum (0.255 and 0.306) in Boris Becker for both the years (Table 6). These results are attributed to the genetic make of the plants as the various genotypes have exhibited a non-consistent response. Kaur (2014) have also found the variations in

Table 5. Effect of different sources of light as night break on partitioning coefficient in Korean chrysanthemum.

\begin{tabular}{|c|c|c|c|c|c|c|c|c|c|}
\hline \multirow{3}{*}{$\begin{array}{l}\text { Sl. } \\
\text { No. }\end{array}$} & \multirow{3}{*}{ Genotype } & \multicolumn{8}{|c|}{ Partitioning coefficient } \\
\hline & & \multicolumn{3}{|c|}{2015} & \multirow[t]{2}{*}{ Mean } & \multicolumn{3}{|c|}{2016} & \multirow[b]{2}{*}{ Mean } \\
\hline & & $\begin{array}{l}\text { Incande- } \\
\text { scent bulb }\end{array}$ & CFL & LED & & $\begin{array}{l}\text { Incande- } \\
\text { scent bulb }\end{array}$ & CFL & LED & \\
\hline 1 & Banglori Local & 24.70 & 25.20 & 26.93 & $25.61^{\mathrm{f}}$ & 25.58 & 26.08 & 27.82 & $26.49^{\mathrm{f}}$ \\
\hline 2 & Boris Becker & 22.60 & 23.33 & 25.67 & $23.87^{\mathrm{g}}$ & 23.55 & 24.29 & 26.62 & $24.82^{\mathrm{g}}$ \\
\hline 3 & Reagan White & 24.00 & 25.03 & 26.50 & $25.18^{\mathrm{f}}$ & 24.88 & 25.92 & 27.38 & $26.06^{\mathrm{f}}$ \\
\hline 4 & Kelvin Mandarin & 39.90 & 39.08 & 39.90 & $39.63^{\mathrm{b}}$ & 40.85 & 40.04 & 40.85 & $40.58^{\mathrm{b}}$ \\
\hline 5 & NBRI Sunil & 24.70 & 27.07 & 30.67 & $27.48^{\mathrm{e}}$ & 25.58 & 27.95 & 31.55 & $28.36^{\mathrm{e}}$ \\
\hline 6 & Reagan Emperor & 30.07 & 29.47 & 28.13 & $29.22^{\mathrm{d}}$ & 31.02 & 30.42 & 29.09 & $30.18^{\mathrm{d}}$ \\
\hline 7 & Otome Pink & 42.20 & 43.67 & 44.93 & $43.60^{\mathrm{a}}$ & 43.08 & 44.55 & 45.82 & $44.48^{\mathrm{a}}$ \\
\hline 8 & Jaya & 19.70 & 19.23 & 20.07 & $19.67^{\mathrm{h}}$ & 20.65 & 20.19 & 21.02 & $20.62^{\mathrm{h}}$ \\
\hline 9 & Punjab Shyamli & 31.17 & 31.43 & 32.57 & $31.72^{\mathrm{c}}$ & 32.05 & 32.32 & 33.45 & $32.61^{\mathrm{c}}$ \\
\hline \multirow[t]{2}{*}{10} & Yellow Delight & 29.30 & 29.90 & 30.43 & $29.88^{\mathrm{d}}$ & 30.25 & 30.85 & 31.39 & $30.83^{\mathrm{d}}$ \\
\hline & Mean & $28.83^{\mathrm{c}}$ & $29.34^{\mathrm{b}}$ & $30.50^{\mathrm{a}}$ & & $29.75^{\mathrm{c}}$ & $30.26^{\mathrm{b}}$ & $31.50^{\mathrm{a}}$ & \\
\hline
\end{tabular}

Table 6. Effect of different sources of light as night break on leaf weight ratio in Korean chrysanthemum.

\begin{tabular}{|c|c|c|c|c|c|c|c|c|c|}
\hline \multirow{3}{*}{$\begin{array}{l}\text { Sl. } \\
\text { No. }\end{array}$} & \multirow{3}{*}{ Genotype } & \multicolumn{8}{|c|}{ Leaf weight ratio } \\
\hline & & \multicolumn{3}{|c|}{2015} & \multirow[b]{2}{*}{ Mean } & \multicolumn{3}{|c|}{2016} & \multirow[b]{2}{*}{ Mean } \\
\hline & & $\begin{array}{l}\text { Incande- } \\
\text { scent bulb }\end{array}$ & CFL & LED & & $\begin{array}{l}\text { Incande- } \\
\text { scent bulb }\end{array}$ & CFL & LED & \\
\hline 1 & Banglori Local & 0.173 & 0.215 & 0.235 & $0.208^{\mathrm{a}}$ & 0.222 & 0.266 & 0.289 & $0.259^{\mathrm{a}}$ \\
\hline 2 & Boris Becker & 0.195 & 0.200 & 0.235 & $0.210^{\mathrm{a}}$ & 0.244 & 0.252 & 0.289 & $0.262^{\mathrm{a}}$ \\
\hline 3 & Reagan White & 0.250 & 0.251 & 0.265 & $0.255^{\mathrm{a}}$ & 0.299 & 0.302 & 0.317 & $0.306^{\mathrm{a}}$ \\
\hline 4 & Kelvin Mandarin & 0.175 & 0.179 & 0.232 & $0.195^{\mathrm{a}}$ & 0.215 & 0.216 & 0.278 & $0.236^{\mathrm{a}}$ \\
\hline 5 & NBRI Sunil & 0.168 & 0.173 & 0.215 & $0.185^{\mathrm{a}}$ & 0.208 & 0.212 & 0.223 & $0.214^{\mathrm{a}}$ \\
\hline 6 & Reagan Emperor & 0.162 & 0.171 & 0.178 & $0.170^{\mathrm{a}}$ & 0.199 & 0.213 & 0.221 & $0.211^{\mathrm{a}}$ \\
\hline 7 & Otome Pink & 0.415 & 0.152 & 0.152 & $0.240^{\mathrm{a}}$ & 0.479 & 0.189 & 0.190 & $0.286^{\mathrm{a}}$ \\
\hline 8 & Jaya & 0.193 & 0.195 & 0.212 & $0.200^{\mathrm{a}}$ & 0.244 & 0.246 & 0.264 & $0.251^{\mathrm{a}}$ \\
\hline 9 & Punjab Shyamli & 0.182 & 0.185 & 0.200 & $0.189^{\mathrm{a}}$ & 0.241 & 0.244 & 0.262 & $0.249^{\mathrm{a}}$ \\
\hline \multirow[t]{2}{*}{10} & Yellow Delight & 0.232 & 0.225 & 0.225 & $0.227^{\mathrm{a}}$ & 0.272 & 0.267 & 0.271 & $0.270^{\mathrm{a}}$ \\
\hline & Mean & $0.215^{\mathrm{a}}$ & $0.195^{\mathrm{a}}$ & $0.215^{\mathrm{a}}$ & & $0.262^{\mathrm{a}}$ & $0.241^{\mathrm{a}}$ & $0.260^{\mathrm{a}}$ & \\
\hline
\end{tabular}

Mean values in each column with the same letter are not significantly different at $\mathrm{p}<0.05$ according to DMRT. *Significant at $\mathrm{p}<0.05$ 
Table 7. Effect of different sources of light as night break on number of stomata per unit area in Korean chrysanthemum.

\begin{tabular}{|c|c|c|c|c|c|c|c|c|c|}
\hline \multirow{3}{*}{$\begin{array}{l}\text { Sl. } \\
\text { No. }\end{array}$} & \multirow{3}{*}{ Genotype } & \multicolumn{8}{|c|}{ Number of stomata per unit area $\left(\mathrm{mm}^{2}\right)$} \\
\hline & & \multicolumn{3}{|c|}{2015} & \multirow[b]{2}{*}{ Mean } & \multicolumn{3}{|c|}{2016} & \multirow[b]{2}{*}{ Mean } \\
\hline & & $\begin{array}{l}\text { Incande- } \\
\text { scent bulb }\end{array}$ & CFL & LED & & $\begin{array}{l}\text { Incande- } \\
\text { scent bulb }\end{array}$ & CFL & LED & \\
\hline 1 & Banglori Local & 52.33 & 43.90 & 52.18 & $49.47^{\mathrm{b}}$ & 52.51 & 44.12 & 52.42 & $49.68^{b}$ \\
\hline 2 & Boris Becker & 50.80 & 52.97 & 56.97 & $53.58^{\mathrm{a}}$ & 50.99 & 53.17 & 57.21 & $53.79^{\mathrm{a}}$ \\
\hline 3 & Reagan White & 51.67 & 47.14 & 57.418 & $52.08^{\mathrm{a}}$ & 49.58 & 55.45 & 56.67 & $53.90^{\mathrm{a}}$ \\
\hline 4 & Kelvin Mandarin & 44.07 & 50.27 & 55.64 & $49.99^{\mathrm{b}}$ & 44.24 & 50.4 & 55.87 & $50.17^{\mathrm{b}}$ \\
\hline 5 & NBRI Sunil & 49.33 & 55.20 & 56.40 & $53.64^{\mathrm{a}}$ & 51.84 & 47.31 & 63.42 & $54.19^{\mathrm{a}}$ \\
\hline 6 & Reagan Emperor & 50.33 & 54.27 & 55.07 & $53.22^{\mathrm{a}}$ & 50.50 & 54.44 & 55.25 & $53.40^{\mathrm{a}}$ \\
\hline 7 & Otome Pink & 46.20 & 55.43 & 57.32 & $52.98^{\mathrm{a}}$ & 46.62 & 55.59 & 57.47 & $53.23^{\mathrm{a}}$ \\
\hline 8 & Jaya & 46.13 & 53.03 & 55.91 & $51.69^{\mathrm{b}}$ & 46.33 & 53.23 & 56.12 & $51.89^{\mathrm{ab}}$ \\
\hline 9 & Punjab Shyamli & 44.00 & 54.33 & 57.85 & $52.06^{\mathrm{b}}$ & 44.18 & 54.52 & 58.05 & $52.25^{\mathrm{a}}$ \\
\hline \multirow[t]{2}{*}{10} & Yellow Delight & 42.17 & 56.67 & 56.00 & $51.61^{\mathrm{b}}$ & 42.40 & 56.89 & 56.23 & $51.84^{\mathrm{a}}$ \\
\hline & Mean & $47.70^{\mathrm{c}}$ & $52.33^{\mathrm{b}}$ & $56.08^{\mathrm{a}}$ & & $47.92^{\mathrm{c}}$ & $52.51^{\mathrm{b}}$ & $56.87^{\mathrm{a}}$ & \\
\hline
\end{tabular}

Mean values in each column with the same letter are not significantly different at $p<0.05$ according to DMRT. *Significant at $\mathrm{p}<0.05$.

Table 8. Effect of different sources of light as night break on anthocyanin content in Korean chrysanthemum.

\begin{tabular}{|c|c|c|c|c|c|c|c|c|c|}
\hline \multirow{3}{*}{$\begin{array}{l}\text { Sl. } \\
\text { No. }\end{array}$} & \multirow{3}{*}{ Genotype } & \multicolumn{8}{|c|}{ Anthocyanins $(\mathrm{mg} / \mathrm{g})$} \\
\hline & & \multicolumn{3}{|c|}{2015} & \multirow[b]{2}{*}{ Mean } & \multicolumn{3}{|c|}{2016} & \multirow[b]{2}{*}{ Mean } \\
\hline & & $\begin{array}{l}\text { Incande- } \\
\text { scent bulb }\end{array}$ & $\mathrm{CFL}$ & LED & & $\begin{array}{l}\text { Incande- } \\
\text { scent bulb }\end{array}$ & CFL & LED & \\
\hline 1 & Banglori Local & ND & ND & ND & $N^{f}$ & ND & ND & ND & $N^{f}$ \\
\hline 2 & Boris Becker & 11.89 & 10.45 & 14.11 & $12.15^{\mathrm{b}}$ & 12.46 & 10.68 & 14.42 & $12.52^{\mathrm{b}}$ \\
\hline 3 & Reagan White & ND & ND & ND & $N D^{f}$ & ND & ND & $\mathrm{ND}$ & $N D^{f}$ \\
\hline 4 & Kelvin Mandarin & 1.45 & 1.61 & 2.69 & $1.92^{\mathrm{d}}$ & 1.63 & 1.81 & 2.80 & $2.08^{\mathrm{d}}$ \\
\hline 5 & NBRI Sunil & ND & ND & $N D^{f}$ & $N D^{f}$ & ND & ND & ND & $N D^{f}$ \\
\hline 6 & Reagan Emperor & 2.13 & 2.09 & 5.08 & $3.10^{\mathrm{c}}$ & 2.45 & 2.30 & 5.22 & $3.32^{\mathrm{c}}$ \\
\hline 7 & Otome Pink & 0.58 & 0.53 & 1.49 & $0.87^{\mathrm{e}}$ & 0.67 & 0.60 & 1.65 & $0.97^{\mathrm{e}}$ \\
\hline 8 & Jaya & 61.44 & 63.41 & 66.08 & $63.64^{\mathrm{a}}$ & 62.50 & 63.96 & 66.93 & $64.46^{\mathrm{a}}$ \\
\hline 9 & Punjab Shyamli & 0.15 & 0.16 & 0.53 & $0.28^{f}$ & 0.17 & 0.17 & 0.62 & $0.32^{\mathrm{f}}$ \\
\hline \multirow[t]{2}{*}{10} & Yellow Delight & ND & ND & ND & $N D^{f}$ & ND & ND & ND & $N D^{f}$ \\
\hline & Mean & $7.76^{\mathrm{b}}$ & $7.83^{\mathrm{b}}$ & $8.99^{\mathrm{a}}$ & & $7.99^{\mathrm{b}}$ & $7.95^{\mathrm{b}}$ & $9.16^{\mathrm{a}}$ & \\
\hline
\end{tabular}

Mean values in each column with the same letter are not significantly different at $\mathrm{p}<0.05$ according to DMRT.

$*$ Significant at $\mathrm{p}<0.05 . \mathrm{ND}=$ Not detected.

these parameters mostly due to genetic constitution of the genotypes under study. The number of stomata per $\mathrm{mm}^{2}$ (Table 7) was found maximum (56.60 and 56.87 per $\mathrm{mm}^{2}$ ) under LED source of light as compared to other two sources of light used for the experiment during 2015 and 2016. Zheng and Van Labeke (2017) have also reported the high stomatal index under different qualities of light from LED source on some ornamental plants. From Tables 8 and 9 it is evident that LED lights were more effective in enhancing the anthocyanins and carotenoids content based on which 
the genotypes can be grouped into high and low anthocyanin and carotenoids, with maximum anthocyanins in genotype Jaya and carotenoids in Boris Becker. These findings are in accordance with the results reported by Mizuno et al. (2011) who have also reported increased anthocyanin and carotenoid contents in cabbage leaves grown under LED lights. Chang et al. (2015) have

Table 9. Effect of different sources of light as night break on carotenoids content in Korean chrysanthemum.

\begin{tabular}{|c|c|c|c|c|c|c|c|c|c|}
\hline \multirow{3}{*}{$\begin{array}{c}\text { Sl. } \\
\text { No. }\end{array}$} & \multirow{3}{*}{ Genotype } & \multicolumn{8}{|c|}{ Carotenoids $(\mathrm{mg} / \mathrm{g})$} \\
\hline & & \multicolumn{3}{|c|}{2015} & \multirow[b]{2}{*}{ Mean } & \multicolumn{3}{|c|}{2016} & \multirow[b]{2}{*}{ Mean } \\
\hline & & $\begin{array}{l}\text { Incande- } \\
\text { scent bulb }\end{array}$ & CFL & LED & & $\begin{array}{l}\text { Incande- } \\
\text { scent bulb }\end{array}$ & CFL & LED & \\
\hline 1 & Banglori Local & 8.16 & 8.64 & 9.65 & $8.82^{\mathrm{h}}$ & 8.48 & 8.94 & 9.96 & $9.13^{h}$ \\
\hline 2 & Boris Becker & 57.21 & 55.45 & 59.97 & $57.54^{\mathrm{a}}$ & 57.51 & 55.77 & 60.28 & $57.85^{\mathrm{a}}$ \\
\hline 3 & Reagan White & 33.11 & 32.84 & 37.91 & $34.62^{\mathrm{d}}$ & 33.34 & 33.10 & 38.16 & $34.87^{\mathrm{d}}$ \\
\hline 4 & Kelvin Mandarin & 6.75 & 6.92 & 8.10 & $7.26^{\mathrm{i}}$ & 6.98 & 7.11 & 8.33 & $7.47^{\mathrm{i}}$ \\
\hline 5 & NBRI Sunil & 8.69 & 9.07 & 11.77 & $9.84^{\mathrm{g}}$ & 8.93 & 9.28 & 11.99 & $10.07^{\mathrm{g}}$ \\
\hline 6 & Reagan Emperor & 36.34 & 38.17 & 40.36 & $38.29^{\mathrm{c}}$ & 36.54 & 38.42 & 40.61 & $38.52^{\mathrm{c}}$ \\
\hline 7 & Otome Pink & 54.61 & 51.88 & 59.28 & $55.26^{\mathrm{b}}$ & 54.83 & 52.09 & 59.51 & $55.48^{\mathrm{b}}$ \\
\hline 8 & Jaya & 5.07 & 5.04 & 6.00 & $5.37^{\mathrm{j}}$ & 5.39 & 5.35 & 6.31 & $5.68^{\mathrm{j}}$ \\
\hline 9 & Punjab Shyamli & 24.77 & 25.27 & 32.22 & $27.42^{\mathrm{e}}$ & 25.18 & 25.66 & 32.64 & $27.83^{\mathrm{e}}$ \\
\hline \multirow[t]{2}{*}{10} & Yellow Delight & 12.79 & 12.77 & 15.84 & $13.80^{\mathrm{f}}$ & 12.96 & 12.96 & 16.07 & $14.00^{\mathrm{f}}$ \\
\hline & Mean & $24.75^{\mathrm{b}}$ & $24.60^{\mathrm{b}}$ & $28.11^{\mathrm{a}}$ & & $25.01^{\mathrm{b}}$ & $24.87^{\mathrm{b}}$ & $28.39^{\mathrm{a}}$ & \\
\hline
\end{tabular}

Mean values in each column with the same letter are not significantly different at $p<0.05$ according to DMRT. *Significant at $\mathrm{p}<0.05$

evaluated large germplasm of chrysanthemum for anthocyanins and carotenoids depicting large differences due to its genetic makeup. Most cultivars that were classified as ND (Non-detectable) or low level showed faint/pale coloured or white coloured petals. It was reported that the biosynthesis of carotenoids could impart white colours to petals in chrysanthemums, since the component was subsequently degraded to colourless compounds by a factor, carotenoid cleavage dioxygenase (Ohmiya 2006). It can be inferred from the two years data that LED lights can be used as an ideal source of light for night break in Korean chrysanthemum to influence the growth patterns which largely contribute to the flower production. From this study, it may be concluded that LED lights used for NB treatment for delay of flowering can effectively influence the growth attributes of Koren chrysanthemum.

\section{References}

Bergstrand KJ and Schussler HK 2012. Growth and photosynthesis of ornamental plants cultivated under different light sources. Acta Hort. 956: 141-47.

Chang HP, Soo CC, Soo-Yun P, Jae KK, Yong JK, Sun OC, Mariad VA, Naif Abdullah Al and San UP 2015. Anthocyanin and carotenoid contents in different cultivars of chrysanthemum (Dendranthema grandiflorum Ramat.) flower. Molecules 20: 11090-11102.

Gardener FP, Pearce RB and Mitchel RL 2003. Physiology of Crop Plants. Scientific Publishers, Jodhpur. 3137.

Kaur P 2014. Regulation of flower production and post harvest keeping quality in chrysanthemum (Chrysanthemum morifolium Ramat). Ph.D. dissertation. Punjab Agricultural University, Ludhiana, India. 62-72. 
Kofranek AM 1992. Cut chrysanthemums. In: Introduction to Floriculture. Larson R A (Ed.), Academic Press, Inc. San Diego, California, U.S.A. 5-42.

Kumar S and Singh M 2017. Effect of photoperiod on growth characteristics in Chrysanthemum morifolium Ramat. cv. Zembla using high pressure sodium light. Res. Crops 18: 110-115.

Massa G, Emmerich J, Morrow R, Bourget C and Mitchell C 2006. Plant Growth lighting for space life support: A review. Gravit Space Biol. 19: 19-29.

Mizuno T, Amaki W and Watanabe H 2011 Effects of monochromatic light irradiation by LED on the growth and anthocyanin contents in laves of cabbage seedlings. Acta Hort. 907: 179-184.

Morrow RC 2008. LED lighting in horticulture. Hort. Sci. 43: 1947-1950.

Ohmiya A, Kishimoto S, Aida R, Yoshioka S and Sumitomo K 2006. Carotenoid cleavage dioxygenase (CmCCD4a) contributes to white colour formation in chrysanthemum petals. Plant Physiol. 142: 11931201.

Sarkka LE, Jokinen K, Ottosen C and Kaukoranta T 2017. Effects of LED and HPS lighting on cucumber leaf photosynthesis, light quality penetration and temperature in the canopy, plant morphology and yield. Agricultural and Food Science 26: 102-110.

Singh R and Bala M 2018. Effect of different sources of light as night break on growth and flowering of Korean Chrysanthemum (Chrysanthemum morifolium Ramat.) Genotypes The Bioscan 13: 653-658

Thakur T and Grewal HS 2019. Growth regulation and off season flowering through night breaks in Chrysanthemum morifolium Ramat. cv Anmol. Bangladesh J. Bot. 48(2): 373-378.

Zheng L and Van Labeke M-C 2017. Long-term effects of red- and blue-light emitting diodes on leaf anatomy and photosynthetic efficiency of three ornamental pot plants. Frontiers in Plant Science 8: $917-$ 921

(Manuscript received on 11 March, 2019; revised on 7 October, 2019) 